\title{
Nuclear reactions studied by quasi-elastic measurements with high precision at backward angles
}

\author{
C. J. Lin ${ }^{\text {a }, ~ H . ~ M . ~ J i a, ~ H . ~ Q . ~ Z h a n g, ~ F . ~ Y a n g, ~ X . ~ X . ~ X u, ~ Z . ~ H . ~ L i u, ~ a n d ~ S . ~ T . ~ Z h a n g ~}$ \\ China Institute of Atomic Energy, P. O. Box 275(10), Beijing 102413, China
}

\begin{abstract}
The excitation functions of quasi-elastic scatterings at backward angles were measured with high precision to study the mechanisms of heavy-ion reactions at near- and sub-barrier energies. The surface diffuseness parameters for the ${ }^{16} \mathrm{O}+{ }^{144,152,154} \mathrm{Sm},{ }^{170} \mathrm{Er},{ }^{174} \mathrm{Yb},{ }^{184,186} \mathrm{~W},{ }^{194,196} \mathrm{Pt}$, and ${ }^{208} \mathrm{~Pb}$ systems have been extracted by the single-channel and coupled-channels calculations, respectively. No anomaly was found in these quasi-elastic processes. The barrier heights and barrier distributions were systematically investigated for these systems. Moreover, the coupled-channels effects of neutron transfers with positive Q-value and the breakup effects of weakly-bound nuclei on the fusion processes were also studied for the ${ }^{32} \mathrm{~S}+{ }^{90,96} \mathrm{Zr}$ systems and the ${ }^{6,7} \mathrm{Li},{ }^{9} \mathrm{Be}+{ }^{208} \mathrm{~Pb}$ systems, respectively.
\end{abstract}

\section{Introduction}

The heavy-ion fusion reactions at near- and sub-barrier energies have been extensively investigated in both experiment and theory for ages. However, large numbers of open questions still remain to be solved, for instance, i) the surface diffuseness anomaly in interaction potential [1-3], ii) the coupled-channels effects of neutron transfers with positive $Q$ value [4-6], iii) the breakup effects of weakly-bound nuclei on the fusion processes [7-10], and so on. Quasi-elastic scatterings and fusion reactions are complementary processes because they undergo the same interaction potential and share the mutual information on the reaction mechanism. Simply speaking, we can use an equation, $T=1-R$, to express this idea. Here $T$ is the transmission probability through a potential barrier, $R$ is the reflection probability by the same barrier and 1 is the total incident flux. Moreover, from the experimental point of view, the detection method is simpler for the quasi-elastic particles at backward angles close to $180^{\circ}$ than that for the fusion residual at forward angles close to beam direction. Therefore, the backward quasi-elastic measurements were employed in experiment to probe the surface diffuseness parameter [11-15], the barrier distribution [16-20], etc., in the past years.

In this paper, recent research activities in our group on the quasi-elastic measurements with high precision at backward angles will be reviewed and discussed. These works concentrated on the surface diffuseness of nuclear potentials, the barrier distributions of neutron transfers with positive Q-value, and the breakup effects of weakly-

a e-mail : cjlin@ciae.ac.cn bound nuclei. The systematic studies of the surface diffuseness parameters of nuclear potentials and the barrier distributions for the ${ }^{16} \mathrm{O}$-induced scatterings, i.e. ${ }^{16} \mathrm{O}+{ }^{144,152,154} \mathrm{Sm},{ }^{170} \mathrm{Er},{ }^{174} \mathrm{Yb},{ }^{184,186} \mathrm{~W},{ }^{194,196} \mathrm{Pt}$, and ${ }^{208} \mathrm{~Pb}$ systems, will be presented in section 2. The details of experimental procedure are included in this section. For the other quasi-elastic measurements, the experimental methods are similar to this. The barrier distributions for ${ }^{32} \mathrm{~S}+{ }^{90,96} \mathrm{Zr}$ systems to study the effects of neutron transfers will be located in section 3 . The breakup effects on fusion processes for the weakly-bound ${ }^{6,7} \mathrm{Li},{ }^{9} \mathrm{Be}+{ }^{208} \mathrm{~Pb}$ systems will described in section 4 . Conclusions will be given in the section 5. Partial of these results mentioned above have been published in Refs. [21-27].

\section{Systematic studies of ${ }^{16} \mathrm{O}$-induced quasi-elastic scatterings}

\subsection{Experimental procedure}

The experiment was performed at the HI-13 tandem accelerator of the Beijing Tandem Accelerator National Laboratory of the China Institute of Atomic Energy (CIAE). A collimated ${ }^{16} \mathrm{O}$ beam with incident energies in the range from 36 to $82 \mathrm{MeV}$ bombarded the targets. The bombarding energies were varied in steps of $3 \mathrm{MeV}$ at the very low energies and in steps of $0.75 \mathrm{MeV}$ at the high energies. The isotopically enriched thin targets (100 - 200 $\mu \mathrm{g} / \mathrm{cm}^{2}$ thickness) of ${ }^{144,152,154} \mathrm{Sm},{ }^{170} \mathrm{Er},{ }^{174} \mathrm{Yb},{ }^{184,186} \mathrm{~W}$, ${ }^{194,196} \mathrm{Pt}$, and ${ }^{208} \mathrm{~Pb}$ were evaporated onto $\sim 20 \mu \mathrm{g} / \mathrm{cm}^{2}$ carbon backings. The target diameters were $3 \mathrm{mms}$. The typical beam spot was $1 \mathrm{~mm}$ but may shift $\pm 1 \mathrm{~mm}$. Two 
collimators with inner diameters of $10 \mathrm{~mm}$ and lengths of $200 \mathrm{~cm}$ were mounted in the entrance and exit tubes about $110 \mathrm{~cm}$ apart from each other. The scattered particles from the reaction targets were detected by four $\mathrm{Si}(\mathrm{Au})$ surface-barrier detectors located at $175^{\circ}$ relative to the beam direction and three $\triangle E-E$ telescopes which consist of a gas ionization chamber and inside three $\mathrm{Si}(\mathrm{Au})$ detectors located at $157.4^{\circ}, 160.8^{\circ}$ and $164.2^{\circ}$ respectively. Four $\mathrm{Si}(\mathrm{Au})$ monitor detectors (up and down, left and right) located at $20^{\circ}$ and $41^{\circ}$ with respect to the beam direction were used to detect the elastic scattering particles for normalization, also used to check the beam quality and to correct the beam direction.

The typical Q-value spectra of the ${ }^{16} \mathrm{O}+{ }^{208} \mathrm{~Pb}$ and ${ }^{184} \mathrm{~W}$ systems at three energies below the barriers are shown in Fig. 1. It can be seen that more exit channels emerge with the increasing energy besides the elastic scattering for the systems, despite of somewhat bad energy resolution. The quasi-elastic events were defined as the sum of the elastic and all the other direct constituents at lower energies in each spectrum. The background counts in the spectra are almost non-existent due to the collimator in the exit tube, which prevents the back-scattering products from the Faraday cup into the backward detectors. After careful analyses, the excitation functions of quasi-elastic scatterings for ${ }^{16} \mathrm{O}+{ }^{144,152,154} \mathrm{Sm},{ }^{170} \mathrm{Er},{ }^{174} \mathrm{Yb},{ }^{184,186} \mathrm{~W}$, ${ }^{194,196} \mathrm{Pt}$, and ${ }^{208} \mathrm{~Pb}$ systems are illustrated in Fig. 2.

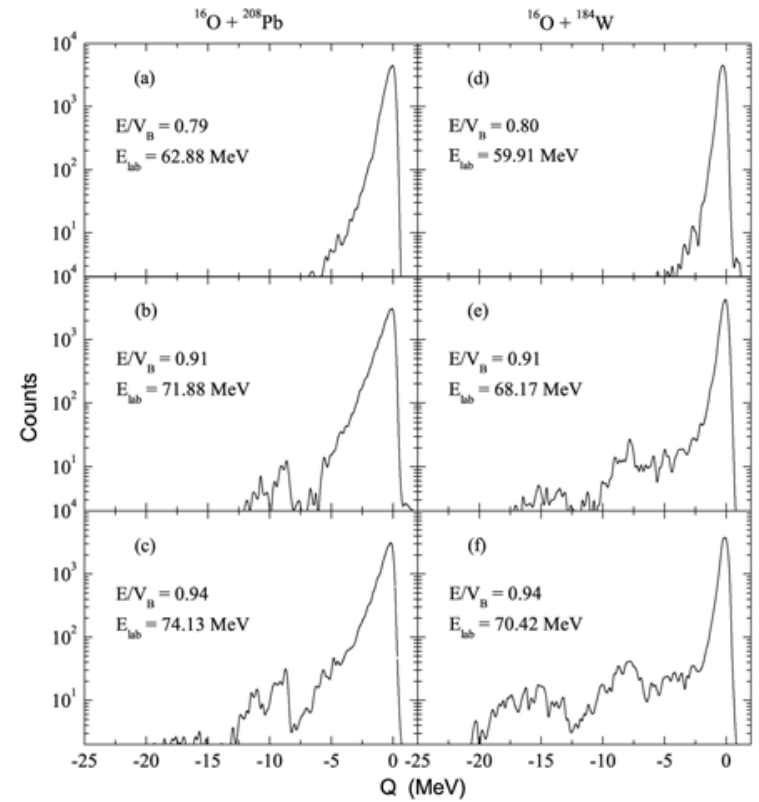

Fig. 1 The Q-value spectra of the ${ }^{16} \mathrm{O}+{ }^{208} \mathrm{~Pb}$ and ${ }^{184} \mathrm{~W}$ systems at three energies below the barriers.

\subsection{Surface diffuseness of nuclear potentials}

The single-channel (SC) and coupled-channels (CC) calculations were performed with a modified version of the code CCFULL [28] for quasi-elastic scattering with a nuclear potential of Woods-Saxon (WS) form. An imaginary potential with $W=30 \mathrm{MeV}, a_{\mathrm{w}}=0.1 \mathrm{fm}$, and $r_{\mathrm{w}}=0.8 \mathrm{fm}$, which was well kept inside the Coulomb barrier, was chosen to represent the rather small absorption from barrier penetration in the calculations. This short-range imaginary potential has no influence on the quasi-elastic scattering cross section predictions because its strength at the surface region is negligible. Considering the real part of the nuclear potential, the surface diffuseness parameter can be determined unambiguously due to the compensative effect of the variations in both the $V_{0}$ and $r_{0}$ values on the Coulomb barrier height energy at the surface region. The parameters of the real part of WS potential were searched for the optimal fitting to the quasi-elastic scattering data. At the same time, the expected energy of the average fusion barrier height should be reproduced in the calculations. The reasonable change of fusion barrier has only little influences on the extracted diffuseness parameters. For consistency, $V_{0}=100 \mathrm{MeV}$ for both single channel and coupled-channels calculations were used for all the reactions. The $r_{0}$ and $a$ values of the real part potential parameters were searched for the best fitting data. In the coupled-channels calculations, the minor difference of the $r_{0}$ parameter due to potential renormalization compared with the single-channel calculation could be omitted because of its insensitivity to the derived diffuseness parameter.

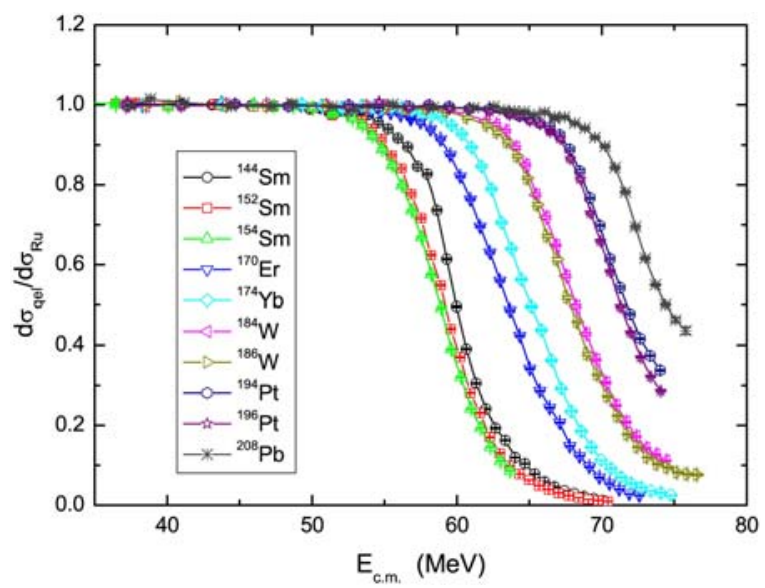

Fig. 2 (color online) Excitation functions of quasi-elastic scatterings for ${ }^{16} \mathrm{O}+{ }^{144,152,154} \mathrm{Sm},{ }^{170} \mathrm{Er},{ }^{174} \mathrm{Yb},{ }^{184,186} \mathrm{~W},{ }^{194,196} \mathrm{Pt}$, and ${ }^{208} \mathrm{~Pb}$ systems.

In the calculations, the couplings to the first $3^{-}$state in ${ }^{16} \mathrm{O}$ only slightly influence the extracted diffuseness parameter. Therefore, the inelastic excitations of ${ }^{16} \mathrm{O}$ were not included in the coupled-channels calculations for all of the measured reactions. Details of the collective states of the target nuclei included in the coupled-channels calculations and the barrier heights extracted by $R=0.5$ in this work (see Sec. 2.3 for details) are given in Table 1. The deformation parameters were deduced by the reduced electromagnetic transition probabilities from the corresponding energy levels, which can be found in the ENSDF library at the website of National Nuclear data Center [29]. Only those experimental data for each reaction, in which the ratios of the quasi-elastic to the Rutherford cross sections are larger than around 0.94, were used in the analyses. Moreover, the counts of these data are larger than $100 \mathrm{k}$, which means the statistical errors are smaller than $0.4 \%$. Fig. 3 shows the experimental data (symbols) as well as the fits by the SC (dashed lines) and $\mathrm{CC}$ (solid lines) calculations for the ${ }^{16} \mathrm{O}+{ }^{154} \mathrm{Sm},{ }^{184} \mathrm{~W},{ }^{196} \mathrm{Pt}$, and ${ }^{208} \mathrm{~Pb}$ systems, respectively. 
Table 1. The excitation energies, configurations, and deformations of the targets as well as the barrier heights extracted by $R=0.5$ in this work.

\begin{tabular}{ccccc}
\hline \hline Target & $\begin{array}{c}E_{\lambda} \\
(\mathrm{MeV})\end{array}$ & $J^{\pi}$ & $\beta_{\lambda}$ & $\begin{array}{c}\mathrm{V}_{\mathrm{B}} \\
(\mathrm{MeV})\end{array}$ \\
\hline${ }^{144} \mathrm{Sm}$ & 1.660 & $2^{+}$ & 0.088 & 59.94 \\
& 1.810 & $3^{-}$ & 0.132 & \pm 0.48 \\
${ }^{152} \mathrm{Sm}$ & 0.122 & $2^{+}$ & 0.307 & 59.19 \\
& 0.36 & $4^{+}$ & 0.137 & \pm 0.50 \\
${ }^{154} \mathrm{Sm}$ & 0.082 & $2^{+}$ & 0.339 & 58.92 \\
& 0.267 & $4^{+}$ & 0.162 & \pm 0.42 \\
${ }^{170} \mathrm{Er}$ & 0.079 & $2^{+}$ & 0.336 & 63.52 \\
& 0.260 & $4^{+}$ & 0.058 & \pm 0.46 \\
${ }^{174} \mathrm{Yb}$ & 0.076 & $2^{+}$ & 0.321 & 65.08 \\
& 0.253 & $4^{+}$ & 0.049 & \pm 0.40 \\
${ }^{184} \mathrm{~W}$ & 0.112 & $2^{+}$ & 0.234 & 68.19 \\
& 0.364 & $4^{+}$ & -0.064 & \pm 0.46 \\
${ }^{186} \mathrm{~W}$ & 0.123 & $2^{+}$ & 0.226 & 67.79 \\
& 0.397 & $4^{+}$ & -0.072 & \pm 0.24 \\
${ }^{194} \mathrm{Pt}$ & 0.328 & $2^{+}$ & -0.143 & 71.71 \\
& 0.811 & $4^{+}$ & -0.038 & \pm 0.96 \\
${ }^{196} \mathrm{Pt}$ & 0.356 & $2^{+}$ & -0.130 & 71.24 \\
& 0.877 & $4^{+}$ & -0.019 & \pm 0.67 \\
${ }^{208} \mathrm{~Pb}$ & 2.615 & $3^{-}$ & 0.111 & 74.40 \\
& 3.198 & $5^{-}$ & 0.058 & \pm 1.01 \\
\hline \hline
\end{tabular}

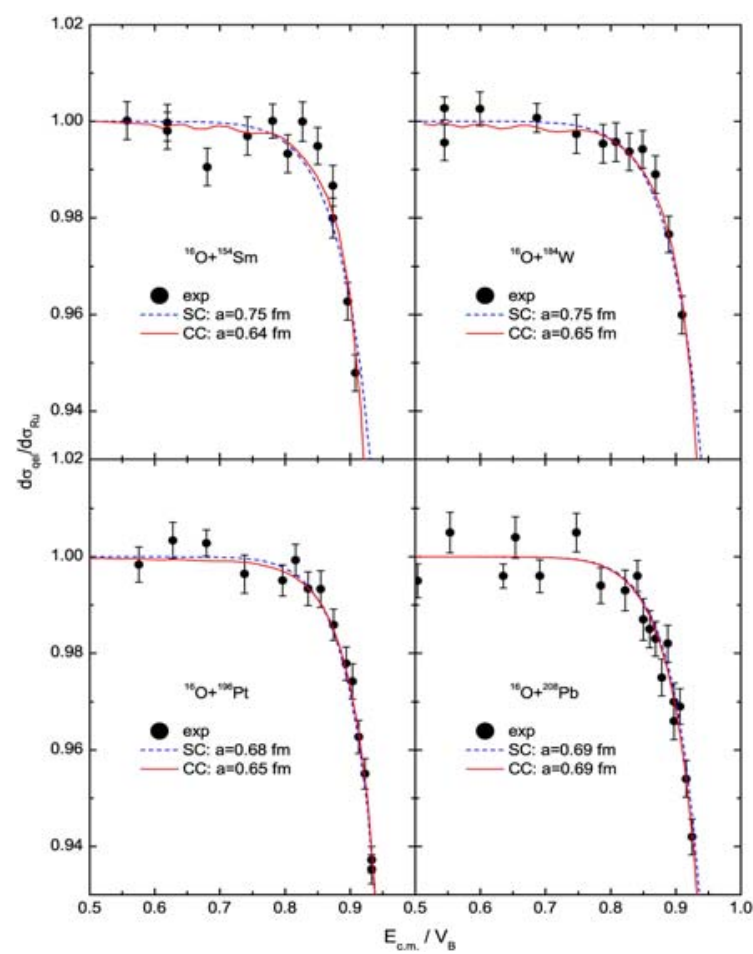

Fig. 3 (color online) The excitation functions with $\mathrm{d} \sigma_{\mathrm{gel}} / \mathrm{d} \sigma_{\mathrm{Ru}}>$ 0.94 as well as the fits by the SC (dashed lines) and CC (solid lines) calculations for the ${ }^{16} \mathrm{O}+{ }^{154} \mathrm{Sm},{ }^{184} \mathrm{~W},{ }^{196} \mathrm{Pt}$, and ${ }^{208} \mathrm{~Pb}$ systems.

Finally, the diffuseness parameters extracted from the $\mathrm{SC}$ and $\mathrm{CC}$ calculations and the fitting goodness for the ${ }^{16} \mathrm{O}+{ }^{152,154} \mathrm{Sm},{ }^{184} \mathrm{~W},{ }^{196} \mathrm{Pt}$, and ${ }^{208} \mathrm{~Pb}$ systems are listed in Table 2. Other systems are still in analysis. The current results show that the $\mathrm{SC}$ fitting quasi-elastic scattering data leads to diffuseness parameter $a$ values in the range from 0.68 to $0.77 \mathrm{fm}$. While the $\mathrm{CC}$ calculations result in a weighted average value for the diffuseness parameter of $a=0.65 \pm 0.01 \mathrm{fm}$ for all the studied reactions. This value agrees with the theoretical calculation result using the double-folding potential model. The effect of couplings influences significantly the quasi-elastic scattering for well-deformed nucleus systems even at deep sub-barrier energies. No anomalous behaviour of a large diffuseness parameter value exists when considering the coupled-channels effect in large-angle quasi-elastic scattering at deep sub-barrier energies.

Table 2. Surface diffuseness parameters and fitting goodness parameters in the $\mathrm{SC}$ and $\mathrm{CC}$ calculations for the ${ }^{16} \mathrm{O}+{ }^{152,154} \mathrm{Sm}$, ${ }^{184} \mathrm{~W},{ }^{196} \mathrm{Pt}$, and ${ }^{208} \mathrm{~Pb}$ systems.

\begin{tabular}{ccccc}
\hline \hline Target & $\begin{array}{c}a^{\mathrm{SC}} \\
(\mathrm{fm})\end{array}$ & $\chi^{2} / N$ & $\begin{array}{c}a^{\mathrm{CC}} \\
(\mathrm{fm})\end{array}$ & $\chi^{2} / N$ \\
\hline${ }^{152} \mathrm{Sm}$ & $0.77 \pm 0.02$ & 1.38 & $0.64 \pm 0.02$ & 1.50 \\
${ }^{154} \mathrm{Sm}$ & $0.75 \pm 0.03$ & 1.91 & $0.65 \pm 0.02$ & 1.21 \\
${ }^{184} \mathrm{~W}$ & $0.75 \pm 0.03$ & 0.63 & $0.65 \pm 0.02$ & 0.58 \\
${ }^{196} \mathrm{Pt}$ & $0.68 \pm 0.02$ & 0.63 & $0.65 \pm 0.02$ & 0.56 \\
${ }^{208} \mathrm{~Pb}$ & $0.69 \pm 0.02$ & 1.38 & $0.69 \pm 0.02$ & 1.21 \\
\hline \hline
\end{tabular}

\subsection{Barrier heights and barrier distributions}

The excitation functions were measured in a wide energy range and the points at $\mathrm{d} \sigma_{\mathrm{qel}} / \mathrm{d} \sigma_{\mathrm{Ru}}=0.5$ have been spanned. Usually the barrier height is determined from the fusion excitation function at the $T=0.5$ point. According to $T=1-R$, the barrier height can also be determined from the backward quasi-elastic excitation function at the $R=0.5$ point. And it can be directly read from Fig. 2 because of $R=\mathrm{d} \sigma_{\mathrm{qe}} / \mathrm{d} \sigma_{\mathrm{Ru}}$. The values of barrier heights determined by $R=0.5$ for all the measured systems are listed in the Table 1 with errors and also plotted in Fig. 4 as a function of the Coulomb parameter $Z$ $=Z_{1} Z_{2} /\left(A_{1}{ }^{1 / 3}+A_{2}{ }^{1 / 3}\right)$. The systematics from Ref. [30] is also shown as dashed line in the figure. It needs to be noted that the systematics was derived from the fusion data. From the figure one can seen that our values are slight larger than the systematic estimations about 0.56 $\mathrm{MeV}$ in average. Further investigations are necessary to explain this difference.

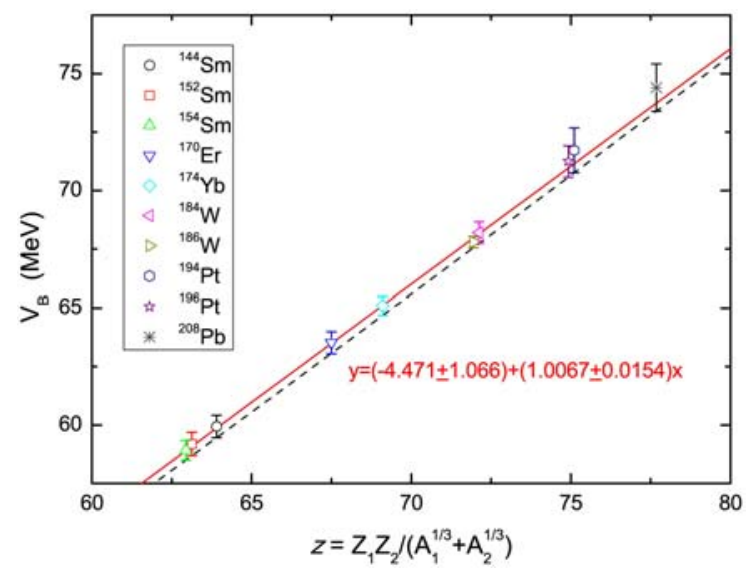

Fig. 4 (color online) Barrier heights determined at the $R=0.5$ points for the ${ }^{16} \mathrm{O}$-induced backward quasi-elastic scatterings. The linear fit to the experimental data is shown as solid line. The systematics from Ref. [30] is also shown as dashed line. 
The barrier distributions can be extracted by the differential of quasi-elastic excitation functions. Because the measured angles were not exactly at $180^{\circ}$, the energies for extracting the barrier distributions should be corrected by the centrifugal energies $[18,20]$. Finally, the extracted barrier distributions of some typical systems are shown in Fig. 5 in order to make a systematic comparison. It is found that for spherical or semi-spherical targets like ${ }^{144} \mathrm{Sm}$ and ${ }^{208} \mathrm{~Pb}$, the barrier distributions are narrower than the deformed targets. Obviously, the widths are relative to the deformation parameters. Besides, the barrier distributions of the heavier systems drop down more quickly than those of the lighter systems at high energies. For example, the distribution terminates at $E / V_{B}$ $\approx 1.05$ for ${ }^{16} \mathrm{O}+{ }^{208} \mathrm{~Pb}$ system, but at $\mathrm{E} / \mathrm{V}_{\mathrm{B}} \approx 1.15$ for ${ }^{16} \mathrm{O}+{ }^{144} \mathrm{Sm}$ system.

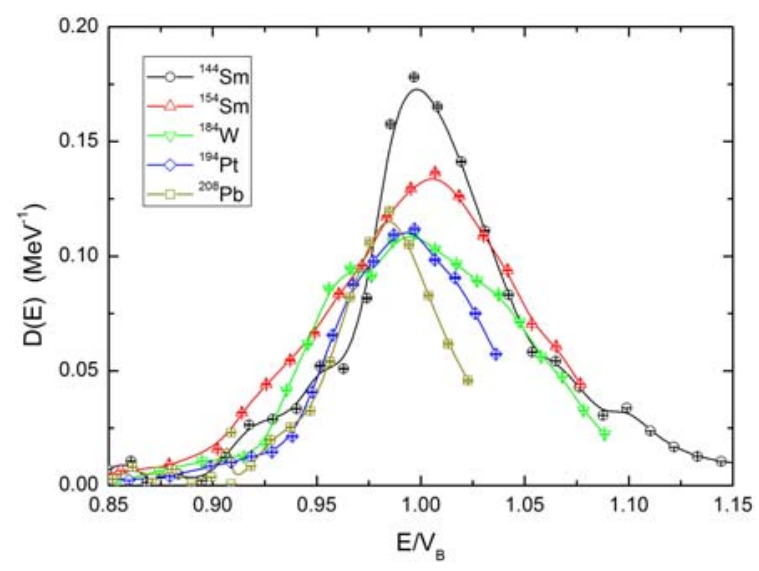

Fig. 5 (color online) Barrier distributions extracted from the ${ }^{16} \mathrm{O}$-induced backward quasi-elastic scatterings.

\section{Effects of neutron transfers with positive Q-values in ${ }^{32} \mathrm{~S}+{ }^{90,96} \mathrm{Zr}$ systems}

The role of neutron transfer or neutron flow in the process of heavy-ion fusion is a topic of current interest. Zagrebaev [31] presented a model incorporating neutron transfer in the coupled-channels (CC) approach and showed that the intermediate neutron transfer channels with positive Q-value really enhance the fusion cross sections at sub-barrier energies. The ${ }^{32} \mathrm{~S}+{ }^{96} \mathrm{Zr}$ system has positive Q-values for $1 n, 2 n, 3 n, 4 n, 5 n$, and $6 n$ neutron transfer channels, while the ${ }^{32} \mathrm{~S}+{ }^{90} \mathrm{Zr}$ system has no any neutron transfers with positive Q-value. Consequently, these two systems are the ideal systems to explore the CC effects of neutron transfers with positive Q-value. The excitation functions of quasi-elastic scatterings were measured at backward angles in the CIAE. Fig. 6 shows the excitation functions of ${ }^{32} \mathrm{~S}+{ }^{90,96} \mathrm{Zr}$ system measured at $\theta_{\mathrm{Lab}}=165.17^{\circ}$ and $154.83^{\circ}$, respectively. The experimental errors include the statistic errors of the event counts only. The relative errors of the cross sections are less than $1 \%$ at low energies and reach to $3 \%$ at high energies. All the error bars are essentially smaller than the data symbols in the figure.

The extracted barrier distributions are shown in Fig. 7. From the figure one can see that the barrier distributions extracted from $165.17^{\circ}$ and $154.83^{\circ}$ are almost identical for both systems. The SC and CC calculations by the CCFULL code are also plotted as the dashed and solid lines, respectively, in the figure. In the $\mathrm{CC}$ calculations, the excited state with $E_{\mathrm{x}}=2.230 \mathrm{MeV}, \beta_{2}=0.32$ in ${ }^{32} \mathrm{~S}$ nucleus, the excited states with $E_{\mathrm{x}}=2.186 \mathrm{MeV}, \beta_{2}=$ 0.09 and $E_{\mathrm{x}}=2.748 \mathrm{MeV}, \beta_{3}=0.22$ in ${ }^{90} \mathrm{Zr}$ nucleus and the excited states with $E_{\mathrm{x}}=1.751 \mathrm{MeV}, \beta_{2}=0.08$ and $E_{\mathrm{x}}$ $=1.897 \mathrm{MeV}, \beta_{3}=0.27$ in ${ }^{96} \mathrm{Zr}$ nucleus were taken into account. The neutron transfer channels were not included in these calculations. Although the nuclear structures of ${ }^{90} \mathrm{Zr}$ and ${ }^{96} \mathrm{Zr}$ are quite similar, the barrier distributions are completely different. The barrier distribution of ${ }^{32} \mathrm{~S}+{ }^{96} \mathrm{Zr}$ system is extremely broader than that of ${ }^{32} \mathrm{~S}+{ }^{90} \mathrm{Zr}$ system, especially in the region of energy below the barrier. It may results from the strong $\mathrm{CC}$ effects of octupole excitations in ${ }^{96} \mathrm{Zr}$ [32]. Furthermore, the multi-neutron transfers with positive Q-values also play important roles at low energies.

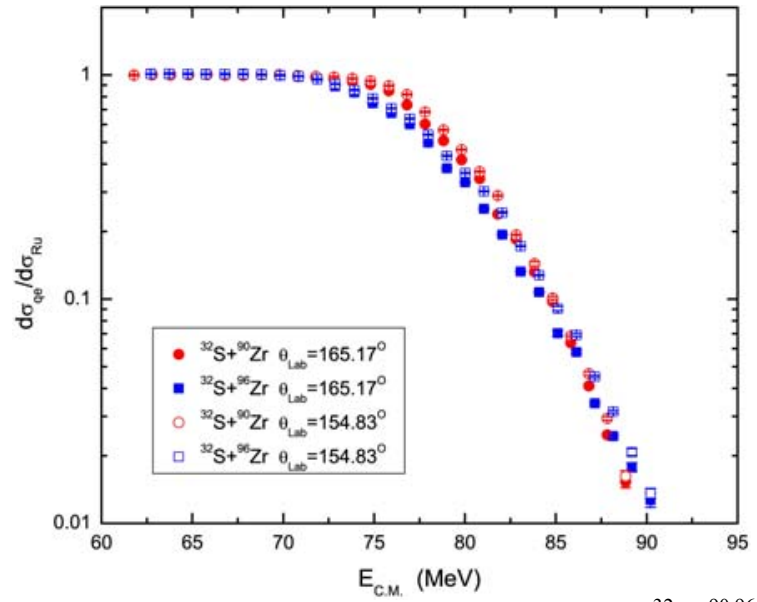

Fig. 6 (color online) The excitation functions of ${ }^{32} \mathrm{~S}+{ }^{90,96} \mathrm{Zr}$ systems measured at $\theta_{\mathrm{Lab}}=165.17^{\circ}$ and $154.83^{\circ}$, respectively versus the centre-of-mass energies.

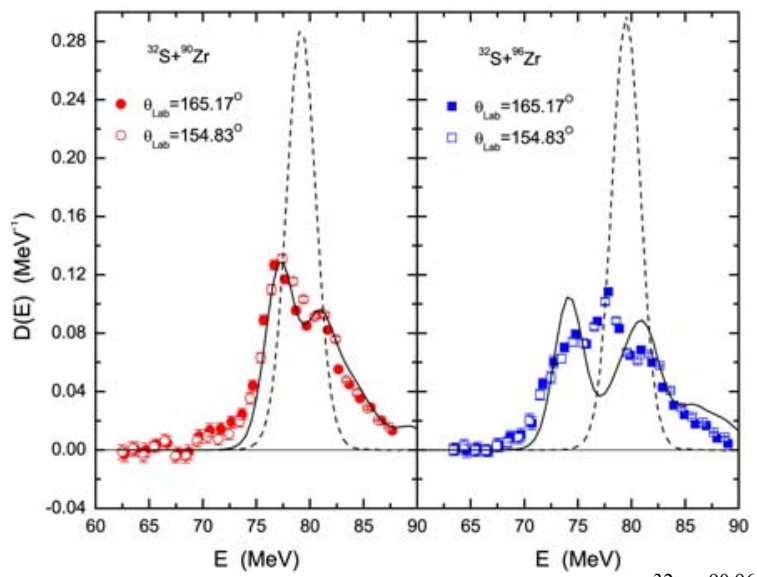

Fig. 7 (color online) The barrier distributions of ${ }^{32} \mathrm{~S}+{ }^{90,96} \mathrm{Zr}$ systems extracted by the quasi-elastic measurements at backward angles. The SC and $\mathrm{CC}$ calculations are also plotted as the dashed and solid lines, respectively.

The excitation functions of fusion evaporation residua for the ${ }^{32} \mathrm{~S}+{ }^{90,96} \mathrm{Zr}$ systems were also measured by an electrostatic deflector [33]. The barrier distributions extracted from these fusion data and the $\mathrm{CC}$ calculations including the neutron transfer channels were presented in Ref. [25] and [34]. 


\section{Breakup effects in ${ }^{6,7} \mathrm{Li},{ }^{9} \mathrm{Be}+{ }^{208} \mathrm{~Pb}$ systems}

Recently, there has been considerable interest in the breakup effects on the fusion and scattering reactions. Particular emphasis is put on the influence of coupling to the breakup channels in the barrier distributions. Interactions of the most weakly bound stable nuclei display some kind of anomalous behaviours, which all attribute to the low-threshold energy for the breakup into their constituents. In fusion of weakly bound nuclei, two independent processes of complete fusion $(\mathrm{CF})$ and incomplete fusion (ICF) occur. The two fusion processes are connected to the dynamics of the projectile fragment. However, it is still difficult to unambiguously predict CF and partial-fusion cross sections. Fortunately, the breakup effects can be studied through the quasi-elastic scatterings at backward angles. As mentioned in the introduction section, fusion is connected to quasi-elastic by $T=1-R$. For the weakly-bound system where the breakup cannot be neglected, the above expression can be modified to $T=1-R-B$, where $B$ stands for the breakup probability during the collision process, which also reduces the incident flux, and $R$ still remains for the quasi-elastic. In this paper, the quasi-elastic includes elastic, inelastic, transfer, and so on, i.e. all the reaction channels except the fusion (or capture for heavier systems) and breakup. The breakup was stripped from the quasielastic in order to solely investigate its effects.

Guiding by the above thought, the quasi-elastic scatterings for the weakly-bound systems ${ }^{6,7} \mathrm{Li},{ }^{9} \mathrm{Be}+{ }^{208} \mathrm{~Pb}$ were measured at backward angles with high precision. Fig. 8 shows the excitation functions of elastic, quasielastic, breakup, and quasi-elastic plus breakup for the ${ }^{6,7} \mathrm{Li}+{ }^{208} \mathrm{~Pb}$ systems. The theoretical calculations by the ECIS code [35] are also shown in the figure for reference. It can be seen that the differential cross sections of the breakup show a maximum around the Coulomb barrier, indicating the breakup is, in substance, a peripheral process like transfer reaction. It also denotes that the Coulomb breakup is the dominant process. The barrier distributions extracted by $T=1-R$ and $T=1-R-B$, respectively, are shown in Fig. 9. The barrier distributions extracted from the $\mathrm{CF}$ data [9] for the neighbour systems of ${ }^{6,7} \mathrm{Li}+{ }^{209} \mathrm{Bi}$ are also illustrated in the figure. One can see that the barrier distributions extracted by $T=1-R-B$ for the ${ }^{6,7} \mathrm{Li}+{ }^{208} \mathrm{~Pb}$ systems are quite similar to those extracted from the $\mathrm{CF}$ data for the ${ }^{6,7} \mathrm{Li}+{ }^{209} \mathrm{Bi}$ systems. It means that the barrier distributions extracted by $T=1-R-B$ and $T=1-R$ from the quasielastic data are equivalent to those extracted from the $\mathrm{CF}$ and ICF data, respectively. Therefore, the breakup effects on fusion reactions can be studied in this way. Similar investigations for the ${ }^{9} \mathrm{Be}+{ }^{208} \mathrm{~Pb}$ system were also done and some results can be found in Ref. [27].

\section{Conclusions}

The excitation functions of quasi-elastic scatterings at backward angles were measured with high precision to study the mechanisms of heavy-ion reactions at near- and

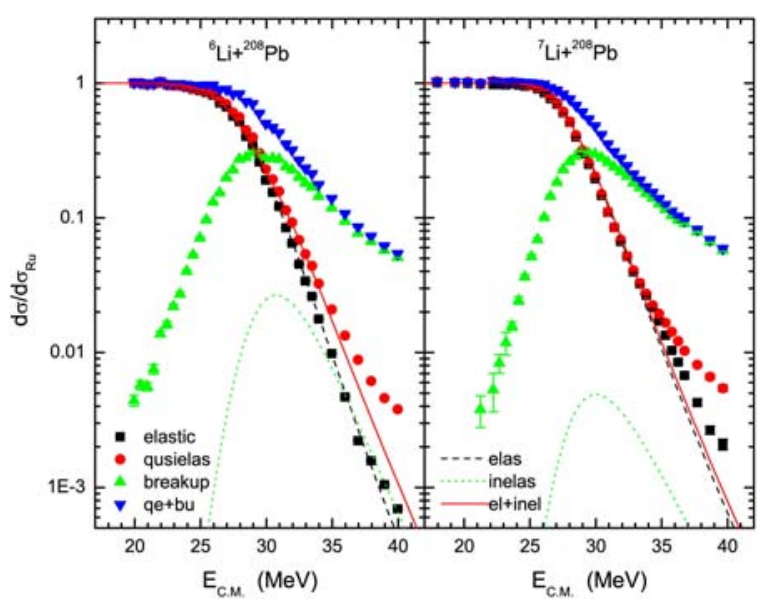

Fig. 8 (color online) The excitation functions of elastic, quasielastic, breakup, and quasi-elastic plus breakup measured at backward angles as well as the theoretical calculations are illustrated for the weakly-bound ${ }^{6,7} \mathrm{Li}+{ }^{208} \mathrm{~Pb}$ systems.

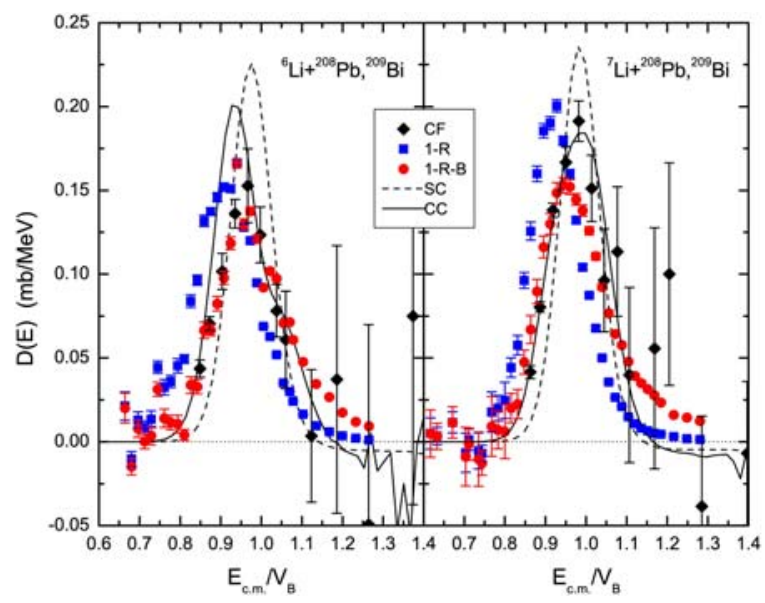

Fig. 9 (color online) The barrier distributions extracted by $1-R$ and $1-R-B$ from the quasi-elastic data for the ${ }^{6,7} \mathrm{Li}^{2}+{ }^{208} \mathrm{~Pb}$ systems and extracted from the $\mathrm{CF}$ data for the ${ }^{6,7} \mathrm{Li}+{ }^{209} \mathrm{Bi}$ systems are plotted as the functions of $E_{\mathrm{c} . \mathrm{m}} / V_{\mathrm{B}}$. The $\mathrm{SC}$ and $\mathrm{CC}$ calculations are also shown in the figure as the dashed and solid lines, respectively.

sub-barrier energies. The surface diffuseness parameters of nuclear potentials for the ${ }^{16} \mathrm{O}+{ }^{144,152,154} \mathrm{Sm},{ }^{170} \mathrm{Er},{ }^{174} \mathrm{Yb}$, ${ }^{184,186} \mathrm{~W},{ }^{194,196} \mathrm{Pt}$, and ${ }^{208} \mathrm{~Pb}$ systems have been extracted by the $\mathrm{SC}$ and $\mathrm{CC}$ analyses in the range of $\mathrm{d} \sigma_{\mathrm{qel}} / \mathrm{d} \sigma_{\mathrm{Ru}}>$ 0.94 . For the $\mathrm{SC}$ analyses, the extracted diffuseness parameters are somewhat larger than the normal value, say $0.65 \mathrm{fm}$, especially for the deformed systems. While the $\mathrm{CC}$ effects were taken into account, these parameters are close to $0.65 \mathrm{fm}$. Therefore, no anomaly was found in these quasi-elastic processes. The barrier heights and barrier distributions were also systematically investigated for these systems and compared with the literature data. For the ${ }^{32} \mathrm{~S}+{ }^{90,96} \mathrm{Zr}$ systems, the barrier distributions extracted by the backward scatterings were compared with those extracted by the fusion reactions. The coupling effects of multi-neutron transfers with positive Q-values and the $3^{-}$vibration states have been confirmed. The barrier distributions which include and exclude the breakup channels, respectively, were extracted for the weakly-bound ${ }^{6,7} \mathrm{Li},{ }^{9} \mathrm{Be}+{ }^{208} \mathrm{~Pb}$ systems. Results show that the barrier distribution is a good probe to investigate the 
$\mathrm{CC}$ effects induced by not only the nuclear structures but also the dynamic processes like transfer and breakup.

\section{Acknowledgments}

This work was supported by the National Natural Science Foundation of China under Grants 10735100, 10727505, and 10975192, and by the Major State Basic Research Developing Program under Grant 2007CB815003.

\section{References}

1. J. O. Newton, C. R. Morton, M. Dasgupta, J. R. Leigh, J. C. Mein, D. J. Hinde, H. Timmers, and K. Hagino, Phys. Rev. C 64, 064608 (2001).

2. J. O. Newton, R. D. Butt, M. Dasgupta, D. J. Hinde, I. I. Gontchar, C. R. Morton, and K. Hagino, Phys. Rev. C 70, 024605 (2004).

3. K. Hagino, T. Takehi, A. B. Balantrkin, and N. Takigawa, Phys. Rev. C 71, 044612 (2005).

4. H. Timmers, L. Corradi, A. M. Stefanini, D. Ackermann, J. H. He, S. Beghini, G. Montagnoli, F. Scarlassara, G. F. Segato, N. Rowley, Phys. Lett. B399, 35 (1997).

5. A. M. Stefanini, F. Scarlassara, S. Beghini, G. Montagnoli, R. Silvestri, M. Trotta, B. R. Behera, L. Corradi, E. Fioretto, A. Gadea, Y. W. Wu, S. Szilner, H. Q. Zhang, Z. H. Liu, M. Ruan, F. Yang, and N. Rowley, Phys. Rev. C 73, 034606 (2006).

6. A. M. Stefanini, B. R. Behera, S. Beghini, L. Corradi, E. Fioretto, A. Gadea, G. Montagnoli, N. Rowley, F. Scarlassara, S. Szilner, and M. Trotta, Phys. Rev. C 76, 014610 (2007).

7. K. Hagino, A. Vitturi, C. H. Dasso, and S. M. Lenzi, Phys. Rev. C 61, 037602 (2000).

8. M. Dasgupta, D. J. Hinde, R. D. Butt, R. M. Anjos, A. C. Berriman, N. Carlin, P. R. S. Gomes, C. R. Morton, J. O. Newton, A. Szanto de Toledo, and K. Hagino, Phys. Rev. Lett. 82, 1395 (1999).

9. M. Dasgupta, D. J. Hinde, K. Hagino, S. B. Moraes, P. R. Gomes, R. M. Anjos, R. D. Butt, A. C. Berriman, N. Carlin, C. R. Morton, J. O. Newton, and A. Szanto de Toledo, Phys. Rev. C 66, 041602R (2002).

10. M. Dasgupta, P. R. S. Gomes, D. J. Hinde, S. B. Moraes, R. M. Anjos, A. C. Berriman, R. D. Butt, N. Carlin, J. Lubian, C. R. Morton, J. O. Newton, and A. Szanto de Toledo, Phys. Rev. C 70, 024606 (2004).

11. K. Washiyama, K. Hagino, and M. Dasgupta, Phys. Rev. C 73, 034607 (2006).

12. L. R. Gasques, M. Evers, D. J. Hinde, M. Dasgupta, P. R. S. Gomes, R. M. Anjos, M. L. Brown, M. D. Rodriguez, R. G. Thomas, and K. Hagino, Phys. Rev. C 76, 024612 (2007).

13. O. A. Capurro, J. O. Fernandez Niello, A. J. Pacheco, and P. R. S. Gomes, Phys. Rev. C 75, 047601 (2007).

14. D. S. Monteiro, J. M. B. Shorto, J. F. P. Huiza, P. R. S. Gomes, and E. Crema, Phys. Rev. C 76, 027601 (2007).

15. M. Evers, M. Dasgupta, D. J. Hinde, L. R. Gasques, M. L. Brown, R. Rafiei, and R. G. Thomas, Phys. Rev. C 78, 034614 (2008).
16. A. T. Kruppa, P. Romain, M. A. Nagarajan, and N. Rowley, Nucl. Phys. A560, 845 (1993).

17. M. V. Andres, N. Rowley, and M. A. Nagarajan, Phys. Lett. B 202, 292 (1988).

18. H. Timmer, J. R. Leigh, M. Dasgupta, D. J. Hinde, R. C. Lemmon, J. C. Mein, C. R. Morton, J. O. Newton, and N. Rowley, Nucl. Phys. A584, 190 (1995).

19. N. Rowley, H. Timmer, J. R. Leigh, M. Dasgupta, D. J. Hinde, J. C. Mein, C. R. Morton, and J. O. Newton, Phys. Lett. B 373, 23 (1996).

20. H. Q. Zhang, F. Yang, C. J. Lin, Z. H. Liu, and Y. M. Hu, Phys. Rev. C 57, R1047 (1998).

21. H. M. Jia, C. J. Lin, H. Q. Zhang, F. Yang, Z. H. Liu, F. Jia, X. X. Xu, S. T. Zhang, and K. Hagino, Nucl. Phys. A834, 189c (2010).

22. C. J. Lin, H. M. Jia, H. Q. Zhang, F. Yang, X. X. Xu, F. Jia, Z. H. Liu, and K. Hagino, Phys. Rev. C 79, 064603 (2009).

23. JIA Hui-Ming, LIN Cheng-Jian, ZHANG Huan-Qiao, LIU Zu-Hua, YANG Feng, JIA Fei, ZHANG ChunLei, AN Guang-Peng, WU Zhen-Dong, XU Xin-Xing, BAI Chun-Lin, and YU Ning, Chin. Phys. Lett. 25, 2834 (2008).

24. F. Yang, C. J. Lin, X. K. Wu, H. Q. Zhang, C. L. Zhang, P. Zhou, and Z. H. Liu, Phys. Rev. C 77, 014601 (2008).

25. H. Q. Zhang, C. J. Lin, F. Yang, H. M. Jia, X. X. Xu, Z. D. Wu, F. Jia, S. T. Zhang, Z. H. Liu, A. Richard, and C. Beck, Phys. Rev. C 82, 054609 (2010).

26. C. J. Lin, H. Q. Zhang, F. Yang, M. Ruan, Z. H. Liu, Y. W. Wu, X. K. Wu, P. Zhou, C. L. Zhang, G. L. Zhang, G. P. An, H. M. Jia, and X. X. Xu, Nucl. Phys. A787, 281c (2007).

27. H. M. Jia, C. J. Lin, H. Q. Zhang, Z. H. Liu, N. Yu, F. Yang, F. Jia, X. X. Xu, Z. D. Wu, S. T. Zhang, and C. L. Bai, Phys. Rev. C 82, 027602 (2010).

28. K. Hagino, N. Rowley, and A. T. Kruppa, Comput. Phys. Commun. 123, 143 (1999).

29. http://www.nndc.bnl.gov/ensdf/

30. W. J. Swiatecki, K. Siwek-Wilczynska, and J. Wilczynski, Phys. Rev. C 71, 014602 (2005).

31. V. I. Zagrebaev, Phys. Rev. C 67, 061601(R) (2003).

32. G. Pollarollo and A. Winther, Phys. Rev. C 62, 054611 (2000).

33. ZHANG Huan-Qiao, LIN Cheng-Jian, YANG Feng, JIA Hui-Ming, ZHOU Ping, AN Guang-Peng, ZHANG Chun-Lei, XU Xin-Xing, Chinese Physics C 34, 1628 (2010).

34. A. Richard, C. Beck, H. Q. Zhang, C. J. Lin, F. Yang, H. M. Jia, X. X. Xu Jia, Z. D. Wu, F. Jia, S. T. Zhang, and Z. H. Liu, oral presentation in the Fusion 11 conference.

35. J. Raynal, Computing as a Language of Physics, (IAEA, Vienna, p. 281, 1972). 